\title{
ANALYSIS OF THE SHORELINE POSITION EXTRACTED FROM LANDSAT TM AND ETM+ IMAGERY
}

\author{
E. Sánchez-García ${ }^{\text {ab, } * \text {, J.E. Pardo-Pascual }}{ }^{\text {ab }}$, A. Balaguer-Beser ${ }^{\text {ac }}$, J. Almonacid-Caballer ${ }^{\text {ab }}$ \\ ${ }^{a}$ Grupo de Cartografía GeoAmbiental y Teledetección, Universitat Politècnica de València, Camino de Vera, s/n \\ 46022, Valencia, Spain \\ ${ }^{\mathrm{b}}$ Departamento de Ingeniería Cartográfica, Geodesia y Fotogrametría, Universitat Politècnica de València, Camino de Vera, s/n \\ 46022, Valencia, Spain - jepardo@cgf.upv.es, (elsncgar, jaialca)@upv.es \\ ${ }^{c}$ Departamento de Matemática Aplicada, Universitat Politècnica de València, Camino de Vera, s/n 46022, Valencia, Spain- \\ abalague@mat.upv.es
}

KEY WORDS: Shoreline position, Landsat imagery, statistical methods, coastal environment, monitoring changes, long-term trends

\begin{abstract}
A statistical analysis of the results obtained by the tool SELI (Shoreline Extraction from Landsat Imagery) is made in order to characterise the medium and long term period changes occurring on beaches. The analysis is based on the hypothesis that intraannual shifts of coastline positions hover around an average position, which would be significant when trying to set these medium and long term trends. Fluctuations around this average are understood as the effect of short-term changes -variations related to sea level, wave run-up, and the immediate morphological beach profile settings of the incident waves- whilst the alterations of the average position will obey changes relating to the global sedimentary harmony of the analysed beach segment. The goal of this study is to assess the validity of extracted Landsat shorelines knowing whether the intrinsic error could alter the position of the computed mean annual shoreline or if it is balanced out between the successive averaged images. Two periods are stablished for the temporal analysis in the area according to the availability of other data taken from high precision sources. Statistical tests performed to compare samples (Landsat versus high accuracy) indicate that the two sources of data provide similar information regarding annual means; coastal behaviour and dynamics, thereby verifying Landsat shorelines as useful data for evolutionary studies.
\end{abstract}

\section{INTRODUCTION}

One of the most characteristic features of beaches is their intense dynamism. The variability of beach spaces -and many coastal environments- can be analysed at different spatial and temporal scales defined by different authors (Carter, 1988; Kraus et al., 1991; Cowell and Thom, 1994; Pye and Blott, 2008) and emphasizing the predominance of certain types of processes and morphological responses examined at each scale level.

Movements in the shoreline position are defined by Kraus et al. (1991) as meso-macro changes. If these succeed during few hours or days will be known as meso changes and, if occur during some years or decades and affect long segments of coast (hundreds of meters or kilometres) will be called macro changes. Certainly, when the scalar perspective is decades, having records of the coastline covering this time range is essential.

The most commonly used data have been the aerial image in which, depending on the study area, clear indicators of the coastline at a particular time are sought. In this study we use the information from Landsat images registered by the TM and ETM+ sensors on the Landsat 5 and Landsat 7 series; the largest useable database of medium resolution images for studying the dynamics of coastal areas. It takes worldwide images since March 1984 every 16 days until November 2011. Moreover, in 2008 the United States Geological Survey (USGS) enabled free access to multiple images with less than $40 \%$ cloud cover, facilitating substantially the possibility of carrying out these kinds of evolutionary works.

The technique called SELI (Shoreline Extraction from Landsat Imagery) used to extract the position of the coastline from Landsat TM and ETM+ imagery is described in Pardo-Pascual et al. (2012), and it detects the boundary between water and land or the wet line. The use of Landsat imagery files as a data source allows us having different coastlines along one year. However, it is essential analysing the level of accuracy that has the obtained shoreline. Pardo-Pascual et al. (2012) analyses the results about 45 images in different artificially stabilized coastal segments by building seawalls. In these places, the impact of wave run-up is null because the water-land limit and the wet line are coincident, which is not true in the case of the beaches. Therefore, it is pertinent to ask whether the deduced waterfront from Landsat images in sedimentary beaches is coincident with a shoreline measured in field or from high-resolution images or, at least, if its employment may provide information with the same validity to characterise the evolutionary trend in medium and long term. Achieving this, and given that unfortunately high precision records are not available at the same time that the Landsat images were taken, the present study compares an annual mean shoreline obtained from high precision data and from Landsat imagery over an area almost no tides.

The basic assumption underlying this proposal is that, as a general rule, intra-annual variations in the coastline position oscillate about an average position which would be the most significant position when trying to set trends. Then, oscillations around that average shoreline are understood as the effect of

\footnotetext{
* Corresponding author. Email: elsncgar@topo.upv.es
} 
changes in short-term while alterations around it, obey changes in the global sedimentary balance of the analysed beach segment.

Testing the accuracy from these extracted annual mean shorelines against other more precise data sources, we evaluate if the inherent Landsat error alters the correct average position or it remains compensated among the successive averaged images in one year. If the study becomes successful, long-term evolutionary trend occurred in a largest coastal sector will be characterised and quantified during the period 1984-2011 through annual average Landsat shorelines.

\section{DATA AND EVALUATION AREA}

Our study area corresponds to the sector B in Figure 1 covering about $19 \mathrm{~km}$ long the Valencian coast concerning to the El Saler beach, south of the port of Valencia. However, data obtained with other high precision sources are only available in the sector A of Figure 1 which covers about $9 \mathrm{~km}$ long. Here is predominant the low and sandy coast along a wide shoal.

From a geomorphological perspective, this coastal strip is part of the barrier island that closed the marshy area where found a lagoon (Albufera). Moreover, unlike what happens in the rest of studied beaches, behind of this appears a large dune field whose formation was subsequent to filler the area with quaternary

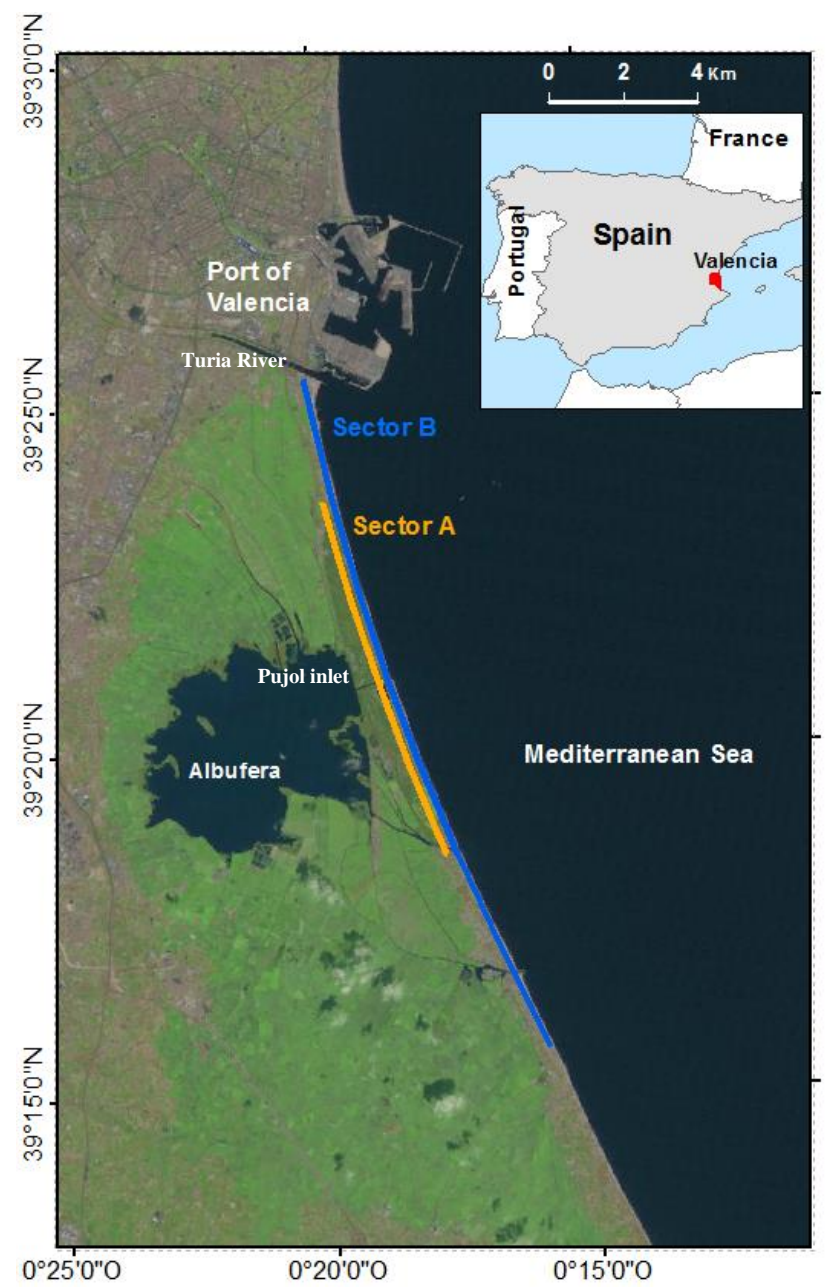

Figure 1. Location map of the two coastal sectors in the Valencian coast. alluvium. According to the incident wave regime, all the area is affected by a clear littoral drift that usually causes a significant southerly sand transport.

This coastal strip, part of the large Gulf of Valencia, has a morphology directly related to the topography of the area and the coast is, in general, low and continuous. These are sandy beaches with a very similar typology which constitute a fairly homogeneous topographical area but with a highly variable wide.

The main difference between sectors A and B is that the first one is all into a natural park with protected coastal dunes, while along the segment outside of sector A, most of these dunes have been substituted by developed areas. The entire evaluation coast has a very small tide regime with typical astronomical sea level variations lower than $0.18 \mathrm{~m}$ although adding meteorological factors these changes can achieve $0.4 \mathrm{~m}$.

Regarding to the used data and for achieving the main goal of this work that is assessing the degree of similarity in the average shoreline between high precision and Landsat sources, we have stablished two different tests on the same area (sector A). The determination of these periods (Figure 2) was merely a consequence of the availability of high precision data. Therefore, the first study covers between October 2006 and November 2007, a total of 372 days and it contains 4 high precision shorelines and 11 from Landsat.

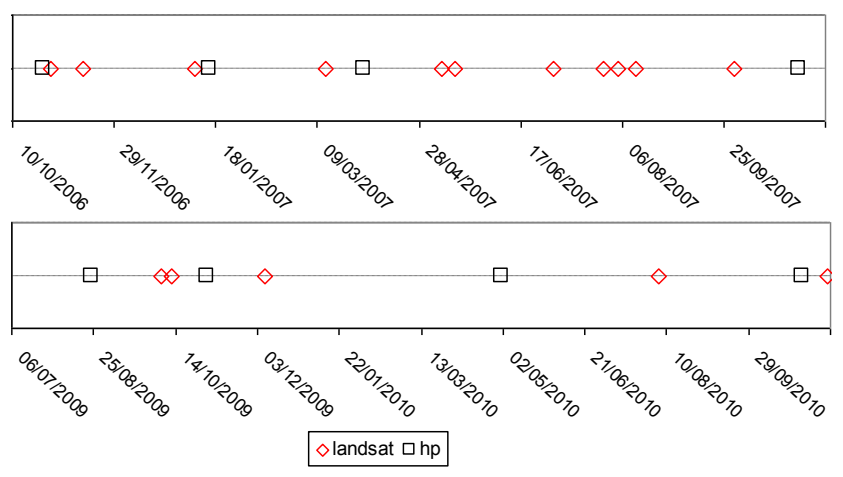

Figure 2. Temporal distribution of high precision and Landsat shorelines during the defined periods: 2006-2007 and 20092010.

The large number of Landsat data consists in a positive point. However, many of these correspond to a summer situation among May and October 2007-, period in which high accuracy data are not available.

The second analysis period spans between August 2009 and November 2010, covering a total of 449 days. It features 4 high precision data and just 5 Landsat but now only two Landsat shorelines are very close in time.

In addition, for analysing the coastal dynamics occurred during a long period of time (1984-2011) in sector B we have a total number of 91 shorelines from Landsat images. Nevertheless, to obviate cyclical trends (intra-annual oscillations) and looking for a robust evolutionary coastal trend analysis, we calculate and work with annual average shorelines

Therefore, we work finally with 15 different average shorelines due to there are empty time slots data (not available Landsat 
images) for the years 1988, 1989, 1991-1998, 2004, 2005 and 2008 (see Figure 3).

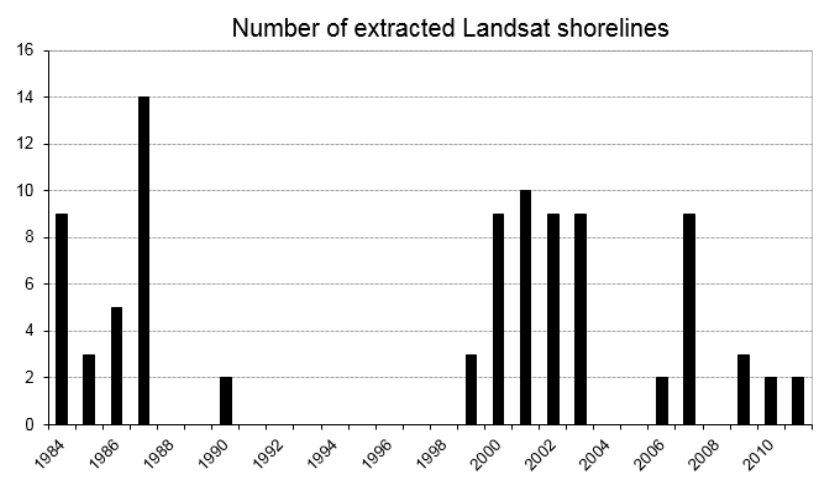

Figure 3. Temporal distribution of high precision and Landsat shorelines during the defined periods: 2006-2007 and 20092010 .

On the one hand, among the coastlines which we called high accuracy, there is one coastline obtained from a LiDAR survey conducted in August 2009 with a density of 2 points $/ \mathrm{m}^{2}$. Available point density and the average slope of the beach, lead to an average error close to $1.67 \mathrm{~m}$ which may occur with this method.

The remaining high precision lines are from direct measurements with a RTK-GPS system. These measurements were performed using a VAT with an RTK-GPS system attached from which successive topographic surveys of the study area were made, taking automatic coordinates records every second (Pardo-Pascual et al., 2011). The precision in these data can be considered less than $1 \mathrm{~m}$. Moreover, these accuracy data have allowed obtaining a DEM for each date to assess the relationship of the slope with the variability of the shoreline position.

On the other hand, the process of extracting shorelines from Landsat has been assessed in Pardo et al. (2012) obtaining an RMSE that ranges from $4.69 \mathrm{~m}$ to $5.47 \mathrm{~m}$. The process considers the automatic extraction of the boundary land-water and the georeferencing coastline system, both with subpixel accuracy. The algorithm provides the shoreline position at separate points every $7.5 \mathrm{~m}$ which we convert to a line using different geometric tolerances in order to reduce angularity and smoothing the final shoreline.

\section{METHODOLOGY}

The structure is composed by three main processes. First of all, obtaining an annual average shoreline; secondly, some statistical tests to compare the behaviour of both samples (high precision and Landsat sources) and finally, a coastal evolutionary study with annual Landsat data.

This task was performed using the Digital Shoreline Analysis System, DSAS (Thieler et al., 2009). The study area is systematically segmented by transects every $25 \mathrm{~m}$ obtaining a total of 403. These ones, start from a baseline located landward and cut the different shorelines perpendicularly. With the intersections between all lines we calculate, for each data set, some statistics such as the average, the median and the standard deviation of the distances in each transect.

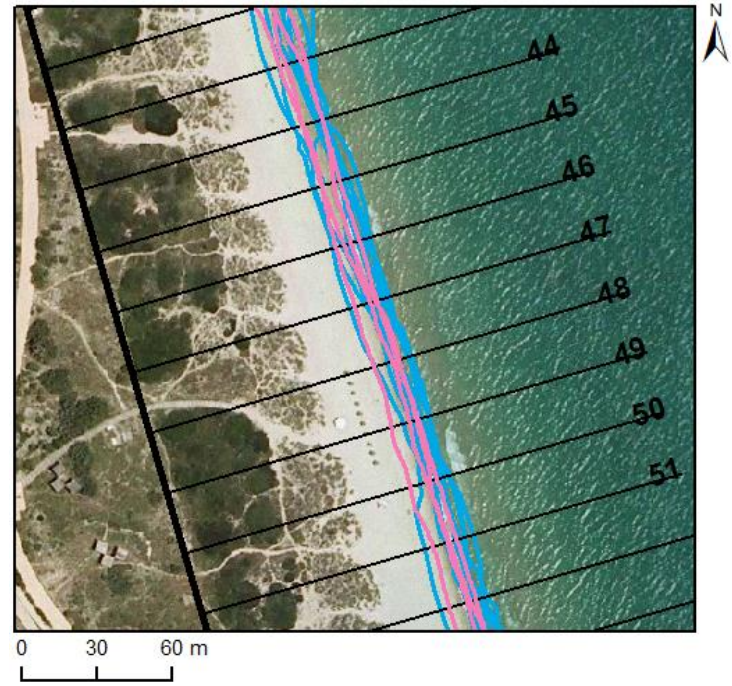

Figure 4. Application example of DSAS. Transects and baseline are shown in black and, in blue and pink, coastlines of Landsat and high precision respectively (studied period 2006-2007).

Then, to determine the grade of similarity between samples (see Figure 5), the t-test is used assuming that the average is a good measure of central tendency. However, their supremacy to detect differences applying t-test to non-normal data is reduced (Bradley, 1968). For this reason, the normality of the data is previously analysed and, in the case that any of the two sets of data (Landsat or high precision) do not follow a normal distribution, we apply the Wilconson Rank-Sum test (Helsel and Hirsch, 2002). It is known that this test has a better control than the classical t-test when the data are contaminated by gross errors (Fay and Proschan, 2010).

For contrasting normality in small samples (size <30), the Shapiro-Wilk test is considered one of the most powerful. It is based upon comparing the quantiles of the fitted normal distribution and the quantiles of the data. Moreover, Yazici and Yolacan, (2007), conclude that for symmetric distributions with small sample sizes, researchers also should choose the Anderson-Darling test of normality. Therefore, we analyse the normal distribution of the data through these tests and considering that the data do not follow a normal distribution when one of the two tests reject the null hypothesis with a confidence of $95 \%$.

In addition, t-test is used to analyse whether the means of two independent samples are different. It is perhaps the most widely used method for comparing two independent groups of data (Helsel and Hirsch, 2002). It is used when the two samples are identically distributed, and by this reason, here it has been applied only on transects where the two data sets, Landsat and HP, follow a normal distribution (95\% confidence). We follow the common strategy which conducts a test on variances prior to the t-test. The problem lies in the difficulty in detecting the equality of variance for the small sample sizes as we are dealing. However, Sawilowsky (2002), proved that conducting the $\mathrm{t}$-test conditioned on the $\mathrm{F}$ test for variances, only resulted in a $5 \%$ loss of power under normality. 


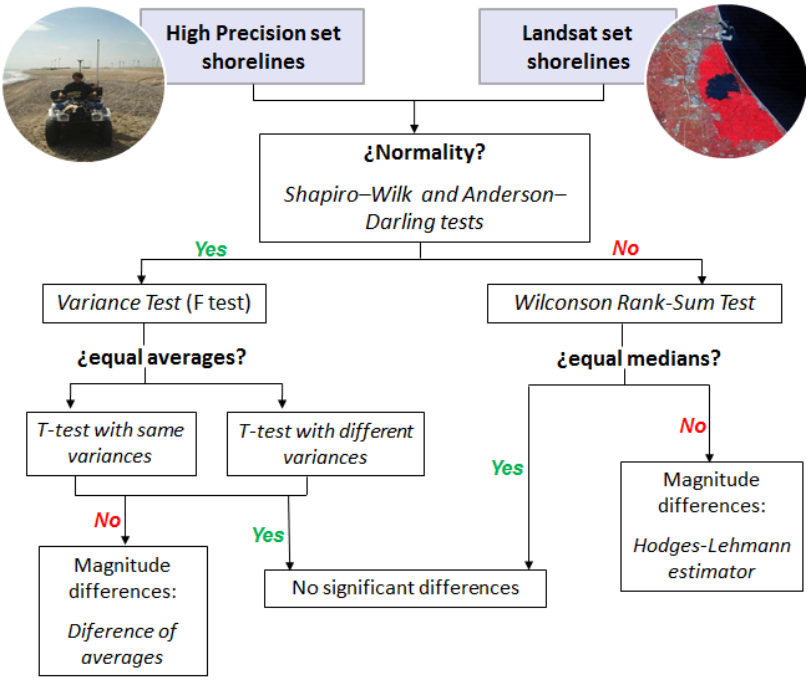

Figure 5. Methodological framework. Comparison process between annual average or median shorelines obtained from Landsat and high precision data.

To sum up, after analyse the t-test (under normality) and RankSum test (in the rest of the cases), we define the whole of transects whose mean positions could be considered as equals. Nevertheless, if the null hypothesis of any test is rejected with a 95\% of confidence level (samples with different average or median position), we evaluate the magnitude of differences between samples. In transects where t-test has been applied and rejected, we compute the difference between averages. In contrast, transects where Rank-Sum test has indicated a significant difference between the medians of the two samples, these are measured with the Hodges-Lehmann estimator.

The last methodological part and, after ensuring the quality of Landsat shorelines for evolutionary studies, we analyse the coastal dynamics occurred during a long period of time (19842011) in sector B using the annual average shorelines previously checked. Carrying out that proposal, again the software DSAS is applied to compute some rate-of-change statistics for the time series of shoreline vector data in transects distributed along the coast each $25 \mathrm{~m}$.

\section{RESULTS}

\subsection{Comparison of samples; period 2006-2007}

High Precision (HP) and Landsat (L) data cover a similar time period, roughly a year. HP data swing from 25/10/2006 to 01/11/2007 and Landsat data from 29/10/2006 to 30/09/2007. There is no HP data from 01/04/2007 until 01/11/2007 whereas the $63.64 \%$ of Landsat data belong to this time interval. Despite the limitations, the differences among both data sets are explored.

According to the methodology described in section 3, first we analyse the percentage of transects that can be adequately modelled by a normal distribution. For this, we have applied the normality tests of Shapiro-Wilk and Anderson Darling, considering that the data do not follow a normal distribution in cases in which one of the two tests reject the null hypothesis with $95 \%$ confidence.
The normality of the data is rejected just in $10.7 \%$ of transects in the case of Landsat data. Since we only count with 4 HP data during the study period, a longer time period has been considered to analyse the normality of these data, from 04/01/2006 until 05/12/2007, in which we have 9 data. The analysis leads to the conclusion that normality thereof is rejected only in the $2.8 \%$ of transects. Moreover, there is only one transect at which we reject the normality of the two sets of data, and there is no stretch of beach over $75 \mathrm{~m}$ length in which the normality assumption of Landsat or HP data is rejected in all transects within it. The difference between averages of Landsat and HP data is greater than $5 \mathrm{~m}$ uniquely in the $14.5 \%$ of transects (figure 6). This percentage drops to $12.94 \%$ in the case of considering only transects with a normal distribution in both groups of data. Notable errors occurring in transects from 66 to 70 and the end of the series transects (starting in number 370 ) in which the average Landsat data becomes more than $8 \mathrm{~m}$ below the average HP data.

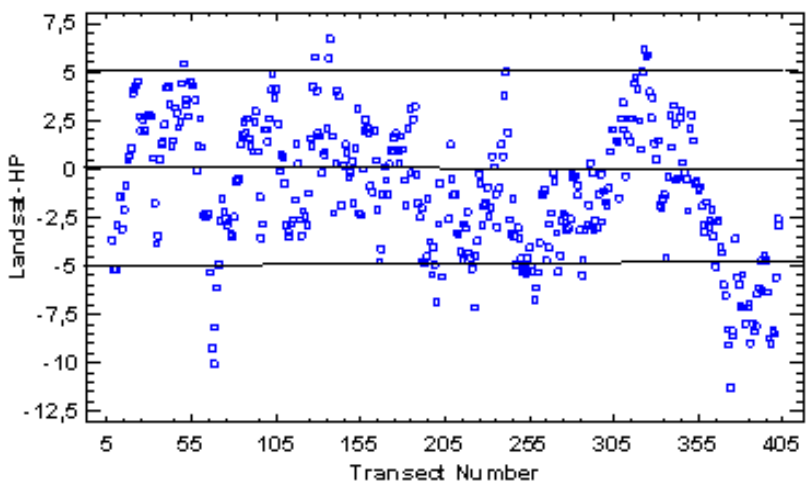

Figure 6. Plot of differences (in meters) between Landsat and HP averages versus the transect number during the period 20062007.

A mean comparison test (t-test) has been performed to analyse differences between Landsat and HP data in transects with a normal distribution. For this purpose, a previous analysis is made to see if two data sets have the same variance. We only reject the hypothesis of equality of variances between Landsat and HP data in the $2.06 \%$ of those transects whose data draw a normal distribution. Moreover, those transects are isolated or forming a group of two adjacent transects.

Therefore, the t-test indicates that we can accept the hypothesis of equality of means with a $95 \%$ of confidence in $96.47 \%$ of transects. Then, analysing the remaining $3.53 \%$, it corresponds with transect numbers $23,50,322,374,383,387,388,389$, 397, 398, 399 and 400 .

Additionally, we observe some transects with a noteworthy difference between Landsat and HP averages (i.e. transects 67, 68 and 70 with differences biggest than $8 \mathrm{~m}$ ). However, these transects have approved the equality of means due to their standard deviation is higher than the overall average whereas, in transects 23,50 and 322 , the standard deviation of the data is well below the average obtained from the whole area (see Table 1) and smaller than the neighbouring transects. This causes them to reject the equality of means with a difference between Landsat and HP average distances close to $5 \mathrm{~m}$.

The Rank Sum test is assessed in transects whose normality is rejected. In this test, in just one transect which is the number 
386, we confirm the no equality of medians with $95 \%$ of confidence. Moreover, the Hodges-Lehmann estimator reaches in this transect a maximum value equal to $-8.725 \mathrm{~m}$.

In order to evaluate how the beach slope affects to the Landsat positioning of mean annual shoreline, an analysis related to the mean slope has been analysed during the period 2006-2007.

In this case, the DEMs acquired from three RTK-GPS surveys made in 2006 (January, April, October) and three more during 2007 (January, April, November), besides the LiDAR survey registered in December 2007, have been used. On every one of these DEMs the slope on the first ten meters measured from the shoreline is calculated. After that, the average and standard deviation of the slope is deduced for each transect (table 1).

The biggest differences of the entire study area between the mean Landsat and HP shoreline position are located since transect 374, being this one where the maximum difference ($11.279 \mathrm{~m}$ ) is reached. We observe that the area which starts from that transect until the end of the sector A, present a terrain slope significantly lower than the average slope of the entire sector. Moreover, since transect 374, the baseline Landsat distances become smaller than HP distances, negative differences which mean that HP shoreline is further offshore than Landsat shoreline.

Hence, evaluating regarding HP sources, we can conclude that the probability of error in Landsat data increases in areas where the terrain slope is smoother.

\begin{tabular}{|c|ccccc|}
\hline Transect & $\begin{array}{c}\text { Difference } \\
(\mathrm{m})\end{array}$ & $\sigma_{\mathrm{L}}$ & $\sigma_{\mathrm{HP}}$ & $\begin{array}{c}\text { Slope } \\
\left({ }^{\circ}\right)\end{array}$ & $\sigma_{\text {Slope }}$ \\
\hline 23 & 4.469 & 2.656 & 3.485 & 4.094 & 0.587 \\
50 & 5.398 & 3.909 & 4.706 & 4.503 & 1.269 \\
322 & 4.992 & 3.750 & 3.909 & 5.184 & 1.302 \\
374 & -11.279 & 6.614 & 4.534 & 3.249 & 1.386 \\
383 & -7.948 & 6.464 & 4.482 & 3.702 & 1.291 \\
386 & -8.725 & & & & \\
387 & -7.941 & 6.460 & 3.922 & 4.892 & 1.691 \\
388 & -8.359 & 4.675 & 8.078 & 3.987 & 1.989 \\
389 & -8.109 & 5.186 & 5.662 & 4.003 & 1.808 \\
397 & -8.747 & 6.448 & 7.025 & 2.630 & 0.778 \\
398 & -8.961 & 5.798 & 7.479 & 2.628 & 1.188 \\
399 & -8.289 & 7.034 & 6.334 & 2.701 & 1.433 \\
400 & -8.478 & 5.731 & 7.353 & 3.593 & 1.352 \\
\hline Average & & & & & \\
(403 & -1.096 & 5.866 & 5.606 & 4.351 & \\
transects $)$ & & & & & \\
\hline
\end{tabular}

Table 1. Results obtained in transects with rejection of equality of means or medians between Landsat and HP data. Slope is related to the changes in the terrain elevation.

\subsection{Comparison of samples; period 2009-2010}

Regarding to the study of the other period, we also have $4 \mathrm{HP}$ data which cover the period from $24 / 8 / 2009$ to $1 / 11 / 2010$ and 5 Landsat data fluctuate from $5 / 10 / 2009$ to $16 / 11 / 2010$. Unlike the previous study, in this period the size of both data sets are similar and data are distributed more uniformly in the timescale. Nevertheless, the reliability of the test of normality is worse because the samples are of size equal or less than 5 .
Then, the t-test has been checked for all transects without distinguishing between those who reject the normality of the data. In that way, we confirm with a confidence of $95 \%$, that in the $92.6 \%$ of transects the means of both sets of data are considered as equals; again, a very encouraging result.

An exemplification of these similitudes among the average shoreline position could be shown in Figure 7. We realise that the difference of the average position described by both sets of data in most transects is minimal. The histogram represents a normal distribution where the differences are distributed equitably around a mean value $(0.357 \mathrm{~m})$ and the $0.357 \pm 3.587 \mathrm{~m}$ covers the $68.3 \%$ of cases. In that way, we are able to assume that the average shoreline position obtained by both sets of data show a nearby behaviour of the coast.

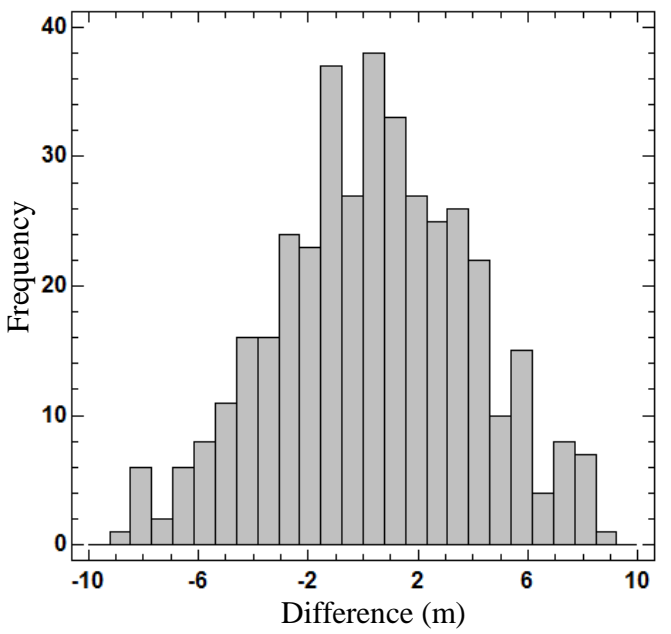

Figure 7. Histogram differences of the average shoreline position along the coast (sector A) defined by both sets of data (high precision and Landsat) and representative of the period 2009-2010.

Analysing the remaining $7.4 \%$ of transects which failed the equality test, we obtain the most relevant differences in transects from number 370 . These are values lower than $-9 m$ in 5 transects of that area and the greatest negative difference -8.8 $\mathrm{m}$ is found in transect number 373. Altogether, there are $18.54 \%$, transects where absolute differences are greater than 5 $\mathrm{m}$ and most of them have a positive difference (Landsat shoreline further offshore than HP shoreline). Recall, that in the period 2006-2007 there was a bias in temporal Landsat data, with a significant percentage of them over a period of time without any data HP, and it does not occur during 2009-2010; factor that could be conditioning the results.

Precision errors in Landsat data cause a larger deviation in the averages when Landsat data are grouped in a small time interval. Thus, it is important that Landsat data are uniformly distributed throughout the time interval in which the average is calculated.

To reach this conclusion has been important consider the proximity of Landsat data and HP, ensuring that both sets of data provide information of similar punctual time values.

\subsection{Evolutionary study (1984-2011) with Landsat data}

One of the applications of Landsat shoreline data focuses on the evolutionary analysis of the coastline in an extended period of time. In this paper, we characterise and quantify the long term 
evolution trend in sector B (a total number of 743 transects systematically distributed every $25 \mathrm{~m}$ alongshore). The average of Landsat data for each calendar year will be used at each transect, which has been computed using the methodology above described.

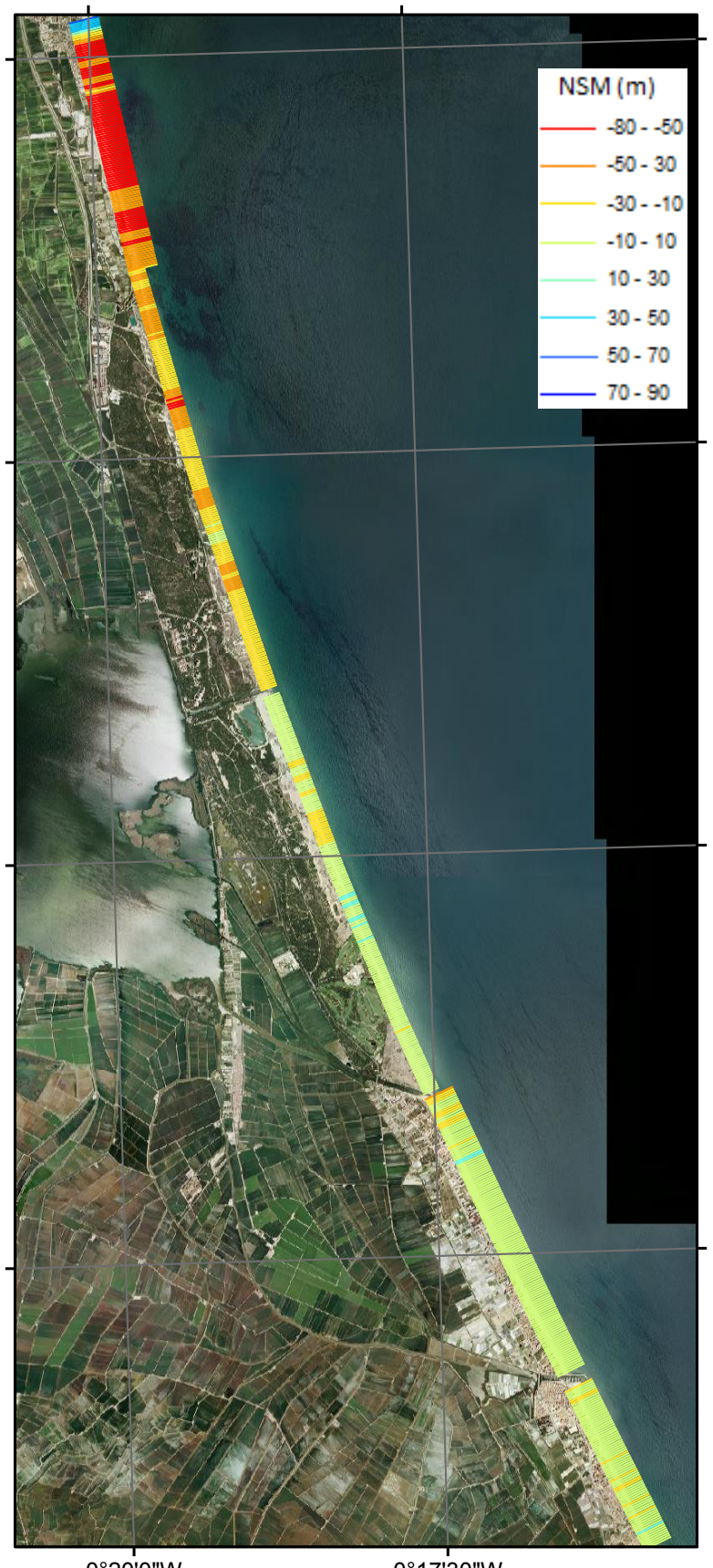

$0^{\circ} 20^{\prime} 0^{\prime \prime} \mathrm{W}$

0 17'30"W

Figure 8. Cartography of the 743 transects covering the sector B and showing the evolutionary trend (Net Shoreline Movement) that have suffered the coast during the period 1984-2011. The smallest width of the central transects distinguishes the sector A, where the quality assessment of Landsat was done, to the evolutionary study in sector B. The information is shown over an orthophoto taken from PNOA sources in 2008.

The statistics obtained with DSAS offer a glimpse of the changes occurring in the waterfront between the years 19842011, consequence of successive mobilizations in a dominant sense which have contributed to the design of the current form of the Valencian coast.

The NSM (Net Shoreline Movement) parameter is defined as the difference between the oldest and the most recent baseline distances of Landsat midlines. Thus, it quantifies the total meters of profit (accretion) or loss (erosion) of sand during the period. Furthermore, the slope or Linear Regression Rate (LRR) of the linear fitting model indicates the meters of change per year due to erosion (negative slope) or accretion (positive slope).

Based on the values taken by these two parameters (NSM and LRR) we can distinguish in Figure 8 three easily identifiable areas. The first ten transects surveyed, about $300 \mathrm{~m}$ from the Turia river mouth, show an average growth of $1.97 \pm 1.2 \mathrm{~m} /$ year, resulting a total earning rate of sand of $46 \mathrm{~m}$ during the 27 years analysed.

These results show the response of different actions that were planned to avoid the expected shadow effect related to the external works of the Port of Valencia (Canalejo and Peña, 1995). Different artificial stiffening actions were made trying to prevent the disappearance of the beach. To get it, an extension of $500 \mathrm{~m}$ in the barrier dam of Turia river mouth was made and also a contribution of $214,000 \mathrm{~m}^{3}$ of sand.

However, the erosion problem has moved southward causing losses of approximately 46 linear meters of sand (NSM average from 10 to 200 group of transects). The most affected area is located two kilometres from the Port of Valencia. Linear regression settings corroborate this erosive trend obtaining a mean value of $-1.77 \pm 0.78 \mathrm{~m} /$ year sand loss.

These results are consequence of the artificial barrier to the long shore sediment transport that is the port. Other factors, such as the quasi null contribution of sediment from a nearby river (the Turia River) should be considered in this sector. The Figure 9 shows the linear regression model for the transect number 75 and indicates the strong erosive tendency experienced between 1984 and 2011 in this zone.

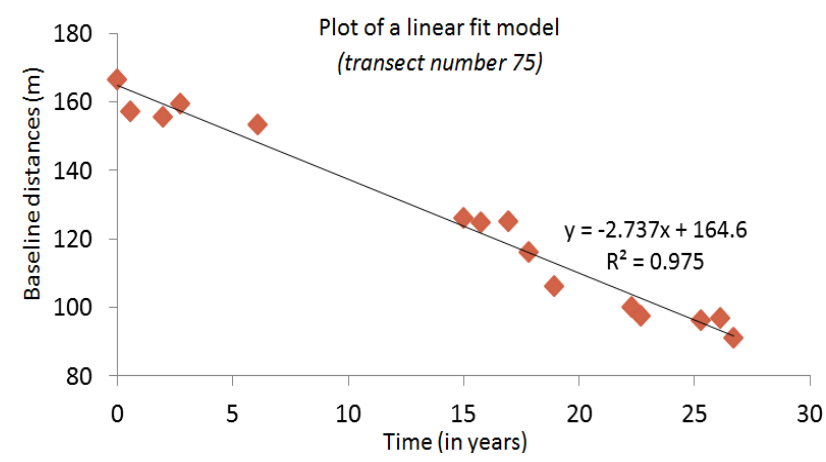

Figure 9. Linear regression fit between baseline distances and time for transect number 75 (numbering concerning the sector B), with Landsat midlines for 1984-2011. Time variable indicates the difference in years from the first value of the time series.

Moreover in the southern zone (from Pujol inlet), transects have NSM and slope values closer to zero (see Figure 8) indicative of minor changes and greater homogeneity. In this area there is an evolutionary trend more stable over time. 
Analysing how the Linear Regression Rate fits in each transect, the NSM is related with it in Figure 10 showing both statistics a similar behaviour along the coast.

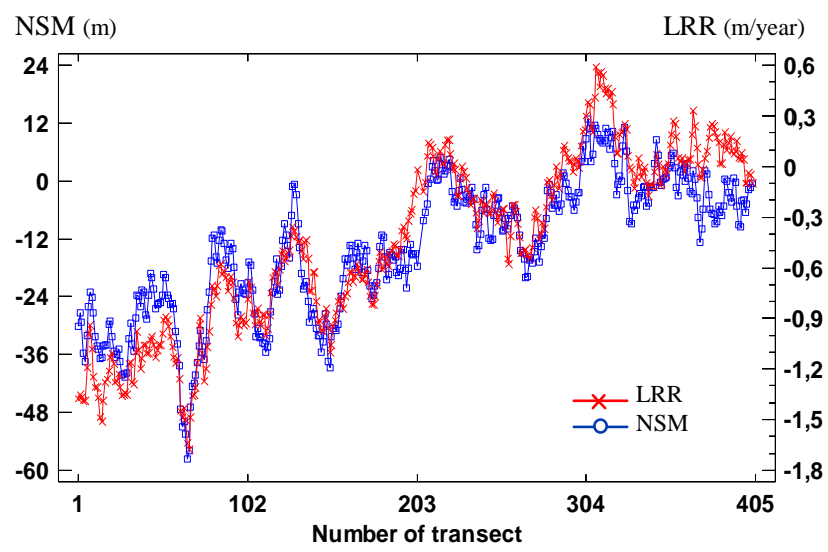

Figure 10. Net Shoreline Movement in m (NSM) and Linear Regression Rate in m/year (LRR). Results obtained with Landsat midlines (1984-2011) for each transect along the sector A.

We realise that the linear model can be used generally among transects with an erosive trend, taking a negative slope in all of them whose R-squared coefficient has an average equal to 0.66 . However there are some exceptions as the example showed in figure 11 (located around 100m north of the Pujol inlet), in which the choice of a second order polynomial may be more appropriate explaining almost $91 \%$ of the variance of data whereas the linear fit represents just $47 \%$ of data.

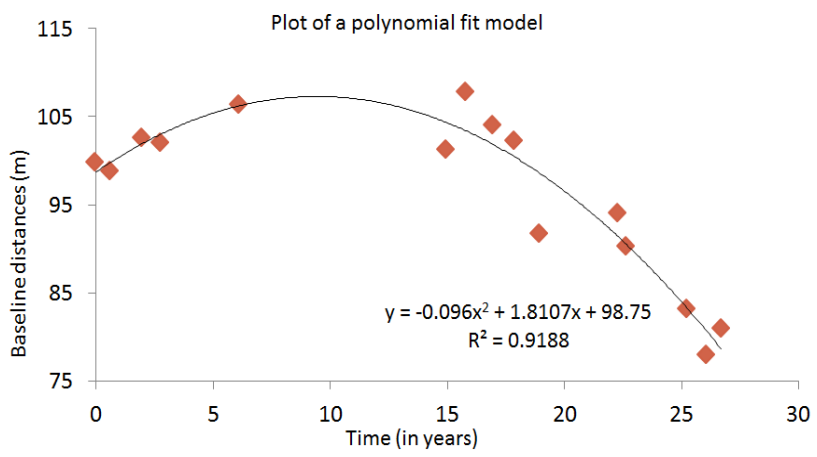

Figure 11. Polynomial regression fit between baseline distances and time for transect number 289 (numbering concerning the sector B) with Landsat midlines for 1984-2011.

Although statistically the regression line is an acceptable model, there is a possible autocorrelation of the residuals, which does not happen when a second order polynomial is fitted. The quadratic polynomial marks a trend change occurred from the year 2001 (time $=17$ from initial time). It indicates a maximum baseline distance in the previous year $(107.69 \mathrm{~m})$ and a minimum in 2010 (77.92 $\mathrm{m}$ regard to baseline). After a slight gain of sediment $(7.84 \mathrm{~m})$ between 1984 and 2000, an increasingly pronounced erosive trend is expected to continue in the coming years. The linear fit has an R-squared equal to 0.8925 when considering only the values of time between 2000 and 2011, avoiding the problem of autocorrelation of the residuals. Therefore, a linear loss of sand may be, in this case, more acceptable than to assume the existence of an acceleration process.
Additionally, in the southern half (in the most transects south of the Pujol inlet) there is no tendency during the period 19842011, being the average of the R-square coefficient in the linear model equal to $0.04 \pm 0.057$. In almost all transect of these zone, there is an accretion process until 2002, with a sharp erosion in 2003. Then, since 2003, the distance to the baseline has been fluctuating around the value obtained in 1984. Consequently, the average of NSM values in this area is equal to $-0.65 \pm 3.7 \mathrm{~m}$ describing a stable coastal behaviour until 2011. However, some minimal changes point to an expected erosive trend coming governed by the port and the data sometimes are better defined by a quadratic polynomial model.

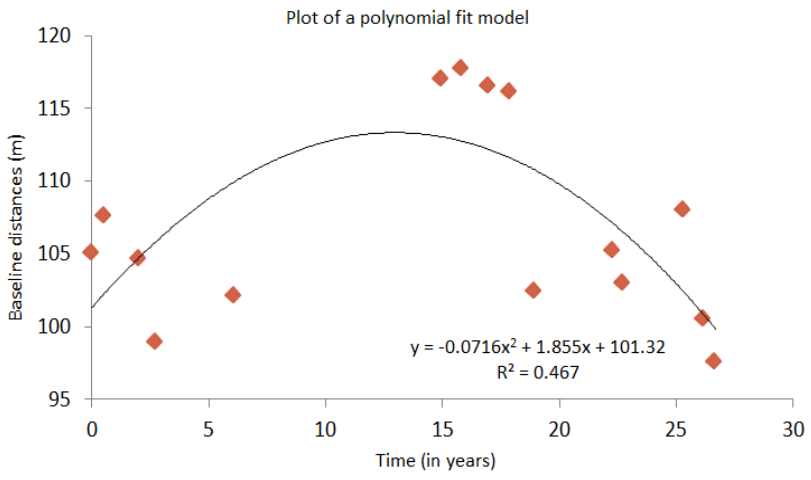

Figure 12. Polynomial regression fit between baseline distances and time for transect number 490 with Landsat midlines for 1984-2011.

\section{CONCLUSION}

The main purpose of many studies carried out around the evolution of coastal areas is to obtain an overview for subsequent prediction and identification of future actions in the context of coastal planning. The study of coastal change is unavoidable when it involves a set of negative implications for their own resources and uses, affecting natural values and socioeconomic interests. In this regard, the methodology used plays a decisive role in the detection and analysis of the magnitude of the changes with accuracy and effectiveness.

Two main objectives have been solved in this paper:

i) On the one hand, the evaluation of the quality and soundness of Landsat data for evolutionary studies has been checked. To achieve it, we tested the application of the methodology described in Pardo-Pascual et al. (2012) to calculate the annual average shoreline on dynamic beaches through information provided by the Landsat satellite data, and comparing the results with those obtained from other, more accurate sources.

The tests performed on two different periods indicate that both sources of data provide similar information verifying the quality of Landsat shorelines.

The results revealed, with a $95 \%$ confidence, the equality of average shoreline positions (obtained by HP and Landsat data) in the $96.47 \%$ and $92.6 \%$ of transects respectively for each analysed period (2006-2007 and 2009-2010). Rejecting the hypothesis of equality of means in less than $8 \%$ of transects and with differences among both sets of data less than $5 \mathrm{~m}$ in more than $80 \%$ transects. This error is similar to the known intrinsic 
error of Landsat data previously assessed in stiffened coastal areas.

Moreover, most transects where the obtained shoreline position by both sets of data present important differences are related with low slope areas -places associated with a higher annual variability of shorelines. The degree of accuracy does not depend on the number of Landsat data, although it improves if these are spaced throughout the studied period and close to the time values of high precision data.

ii) On the other hand, the evolutionary analysis carried out along a coastal area with annual average Landsat data describes the medium and long term shoreline changes occurring during the period 1984-2011. Minimizing the intra-annual oscillations in a year, and working with annual average shorelines, is instrumental for assessing long-term trends.

In a general framework of sedimentary dearth and recessionary trend (strongly associated with changes in port structures and a considerable reduction of sediment inputs), ancient shores of accumulation have become areas under the dominance of erosive processes.

The adjacent beaches to the port and before the Pujol inlet are the most affected by the dams of the port of Valencia where a regression of the beach appears as a widespread pattern. Despite the fact that the erosive wave seems to spread southward, the beaches after Pujol inlet form a more stable coastal sector with some slight events of sedimentary accretion.

\section{ACKNOWLEDGEMENTS}

This work is part of the $\mathrm{PhD}$ of the first author which is supported by the "Ministerio de Educación, Cultura y Deporte" of Spain (state program in I+D+i 2013-2016).

\section{REFERENCES}

Bradley, J.V., 1968. Distribution-Free Statistical Tests. Prentice-Hall, Englewood Cliffs, NJ, pp. 388.

Canalejo, P., Peña, C. 1995. Regeneración de la playa de Pinedo (Valencia). En ingeniería del agua, Ministerio de Obras Públicas, Transporte y Medio Ambiente (MOPTMA), pp. 209.

Carter, RWG., 1988. Coastal environments. An introduction to physical, ecological and cultural systems of coastlines. Accademic Press, pp. 617.

Cowell, PJ, Thom, BG., 1994. Morphodynamics of coastal evolution. In Carter, RWG and Woodroffe, CD (eds.): Coastal evolution: an introduction. Cambridge Univeristy Press, Cambridge, pp. 33-86.

Fay, M.P., Proschan, M.A., 2010. Wilcoxon-Mann-Whitney or t-test? On assumptions for hypothesis tests and multiple interpretations of decision rules. Statistics Surveys, 4, pp. 1-39, DOI: 10.1214/09-SS051

Helsel, D.R., Hirsch, R.M., 2002. Statistical Methods in Water Resources. U.S. Geological Survey Techniques of Water Resources Investigations. Book 4, Chapter A3. Publication available at: http://water.usgs.gov/pubs/twri/twri4a3/.
Kraus, N.C., Larson, M., Kriebel, D.L., 1991. Evaluation of beach erosion and accretion predictors. Coastal Sediments '91, American Society of Civil Engineers, Seattle, pp. 527-587.

Pardo-Pascual JE., Almonacid-Caballer J, Ruiz LA., PalomarVázquez J.,2012. Automatic extraction of shorelines from Landsat TM and ETM multi-temporal images with subpixel precision. Remote Sensing of Environment 123, pp. 1-11.

Pardo-Pascual JE., Palomar-Vázquez JM., García-Asenjo Villamayor L., Garrigues P., 2011. Determinación de la tendencia evolutiva en un segmento de playa basándose en múltiples levantamientos tridimensionales. Avances en Geomorfología Litoral, VI Jornadas de Geomorfología Litoral, Tarragona, pp. 493-496.

Pye, K., and Blott, S.J., 2008. Decadal-scale variation in dune erosion and accretion rates: an investigation of the significance of changing storm tide frequency and magnitude on the Sefton coast, UK. Geomorphology 102, pp. 652-666.

Sawilowsky, S.S. (2002). Fermat, Schubert, Einstein, and Behrens-Fisher: The Probable Difference Between Two Means When $\sigma 1 \neq \sigma 2$. Journal of Modern Applied Statistical Methods 1 (2), pp. 461-472.

Thieler ER, Himmelstoss EA, Zichichi JL, Ayhan E. 2009. Digital Shoreline Analysis System (DSAS) version 4.0 An ArcGIS extension for calculating shoreline changes. U.S. Geological Survey Open-File Report 2008-1278.

Yazici, B., Yolacan, S., (2007). A comparison of various tests of normality, Journal of Statistical Computation and Simulation Vol. 77, No. 2, February 2007, 175-183 